\section{Heterogeneity in genomic disorders}

A previous study (Nat. Genet. 42, 203-209, 2010) suggested a 'two-hit' model for the severity of developmental delay in individuals with a microdeletion at chromosome 16p12.1. Now, Evan Eichler and colleagues expand the two-hit model to a broader genomic context (N. Engl. J. Med. 367, 1321-1331, 2012). They performed array-comparative genomic hybridization (aCGH) analysis of 32,587 children with developmental delay. As a control set, they analyzed 8,329 individuals who did not display overt neurological disorders. They identified 2,312 children with developmental delay who carried 1 of 72 rare copy-number variants (CNVs), most of which have been associated with intellectual disability. Of the 2,312 children, 200 also harbored an additional large autosomal CNV. The authors observed an enrichment of second-site hits in children with a phenotypically variable genomic disorder, supporting the hypothesis that additional genetic variants contribute to phenotypic variation. Information on the mode of inheritance was available for $\mathbf{4 6}$ children with first- and second-site CNVs, which indicated that 33 of 46 of the second-site variants were inherited. Finally, the authors analyzed detailed clinical data for 161 children (96 with a first-site variant and 65 with multiple variants) and found a qualitative increase in deficits in those with multiple variants.

\section{Rare-variant association methods}

Several methods for aggregate rare-variant association testing have recently been reported, including collapsing or weighting methods and gene- or region-based association tests. Although it is possible to estimate the average genetic effect for a group of rare variants from aggregate tests, there are potential biases, including winner's curse, selection procedures and differences between populations. Suzanne Leal and Dajiang Liu now report a new method to correct for bias in estimating the average genetic effect of a group of rare variants jointly analyzed for association consisting of a resampling-based approach and a bootstrap-sample-split algorithm (Am. J. Hum. Genet. 91, 585-596, 2012). They compare methods for estimating the average genetic effect and variance across a range of models in simulations, finding that the estimated variance is always less than the true locus-specific genetic variance, due to the inclusion of non-causal variants as well as causal variants with heterogeneous effects. The authors report the application of the new method to a resequencing data set of 4 genes in 1,045 individuals from the Dallas Heart Study, testing rare-variant associations with metabolic quantitative traits. The authors demonstrate the efficient estimation of average genetic effects in joint analysis of rare variants and note that estimated variance should be considered as a lower bound for the locus-specific variance.

$O B$

\section{Potential Parkinson disease and ALS drug}

The aminopropyl carbazole compound P7C3 has previously been reported to have proneurogenic, neuroprotective properties in adult mice. Now, in two papers, Andrew Pieper and colleagues report the neuroprotective effects of a variant of $\mathrm{P} 7 \mathrm{C} 3$ (P7C3A20) in mouse models of amyotrophic lateral sclerosis (ALS) and Parkinson disease. Tesla et al. (Proc. Natl. Acad. Sci. USA, published online 1 October 2012; doi:10.1073/ pnas.1213960109) show that administration of P7C3A20 at the time of disease onset in G93A-SOD1 mutant mice (a mouse model of ALS) provides significant protection from spinal motor neuron cell death. After about 2 weeks of treatment, P7C3A20-treated mice displayed improved rotarod performance. Significant improvements were also observed in walking gait. In another paper, the authors used toxin-induced models of Parkinson disease, including a model in which mice are exposed to the potent, selective toxin 1-methyl-4-phenyl-1,2,3,6-tetrahydropyridine (MPTP) that kills dopaminergic neurons. De Jesús-Cortés et al. report that P7C3A20 confers protection from MPTP-induced neuronal cell death in mice (Proc. Natl. Acad. Sci. USA, published online 1 October 2012; doi:10.1073/pnas.1213956109). The authors also found that P7C3A20 blocks 1-methyl-4-phenylpyridinium $\left(\mathrm{MPP}^{+}\right)$-induced dopaminergic neuronal death in Caenorhabditis elegans. $\mathrm{MPP}^{+}$-exposed worms treated with P7C3A20 also showed improvements in locomotion. The two studies suggest that further work on P7C3A20 or another P7C3 variant may lead to effective treatments for neurodegenerative diseases.

$P F$

\section{Sensing flow at the node}

The establishment of left-right organ asymmetry in vertebrates requires leftward flow generated by motile cilia at the embryonic node, but the precise mechanism by which flow triggers asymmetric signaling pathways has remained unclear. Petra Pennekamp, Hiroshi Hamada and colleagues (Science, published online 13 September 2012; doi:10.1126/ science.1222538) now provide strong experimental evidence that mechanosensory cilia located in the perinodal region have a key role in establishing the vertebrate left-right axis. Using a series of elegant transgenic models coupled with an experimental system for generating artificial flow across the node region, they show that $\mathrm{Pkd} 2$, which has previously been implicated in cilia-dependent mechanosensation in kidney cells, is specifically required in crown cells located at the periphery of the node to sense directional flow and initiate downstream signaling events. They further show that the ability of crown cells to respond to flow is dependent on ciliary localization of Pkd2. Additional work suggests that this $\mathrm{Pkd} 2$-dependent flow sensing results in elevated calcium signaling in crown cells on the left side of the node, which initiates the asymmetric patterns of gene expression responsible for specifying organ asymmetries.

KV

\section{Admixed genomes}

Carlos Bustamante and colleagues demonstrate the potential of using local ancestry analysis of the genome sequences of admixed individuals for improved demographic inference (Am. J. Hum. Genet. 91, 660-671, 2012). The authors examined the wholegenome sequences of 50 individuals from 11 diverse populations. Their initial comparisons showed expected patterns, including an average of $18 \%$ greater genetic variation in genomes from African individuals and more rare variants than expected under a standard neutral model. They further examined the genome sequences of 12 individuals with recent admixed ancestry. Using the PCAdmix algorithm to analyze each chromosome and partition genomic regions by inferred European, African or Native American ancestry, they were able to assign inferred local ancestry to $79-95 \%$ of each genome with an accuracy of $80-99 \%$. They modeled the distribution of ancestry-tract lengths and found departures from predictions of a pulse admixture model. A continuous or repeated migration model provided improved fit to genetic and historic data, including continuous gene flow from Europe. The authors estimate that $70 \%$ of the European ancestry in modern AfricanAmericans dates to European gene flow 7-8 generations ago. They also highlight the need for new methods for analyzing admixed genomes. 